\title{
The BRS Method and Geometric Quantization: Some Examples
}

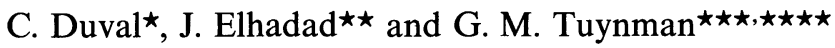 \\ CPT-CNRS, $\star \star \star \star \star$ Luminy, Case 907, F-13288 Marseille Cedex 9, France
}

\begin{abstract}
We recall the method of quantization of constraints as outlined by Kostant and Sternberg. We discuss various examples to test compatibility between geometric quantization and this procedure. In almost all cases reduction and quantization commute.
\end{abstract}

\section{Introduction}

Kostant and Sternberg have proposed in their paper [KS] a very general scheme to quantize a reduced system by using BRS symmetry. However they make no choice of a specific quantization method. In this paper we test in some examples their procedure using geometric quantization.

Although the procedure itself can be stated in quite simple terms, we nevertheless give a lengthy theoretical survey because it contains a non trivial-modification of the Dirac quantization of constraints [Dir]. Moreover in the first part of our paper we point out a series of technical problems which can be related to the $\mathrm{KS}$ method.

Our motivation in dealing with this procedure is to see whether some problems and difficulties encountered in geometric quantization can be solved in this way-notably the problems found in the quantization of spin-spin interaction Hamiltonians and those related to the quantization of the geodesic flow. We should mention that our examples (and the "theory") treat only the special case in which the reduction from the original (finite dimensional) phase space to the reduced one is given by a free action of a Lie group and in which the constrained manifold is coisotropic (only first class constraints). Our examples do not comply with the conditions found in Gotay's paper [Got] where sufficient conditions are given for geometric quantization and reduction to commute. In particular we do not require

* Université d'Aix-Marseille II \& Centre de Physique Théorique

$\star \star$ Université de Provence \& Centre de Physique Théorique

$\star \star \star$ MSRI, 1000 Centennial Drive, Berkeley, CA 94720, USA

$\star \star \star \star$ Supported by the Dutch organization for Scientific Research (NWO)

$\star \star \star \star \star$ Unité Propre de Recherche 7061, CNRS 
the group to admit a bi-invariant metric nor the action to be a lifted cotangent action.

The paper is organized as follows:

- In the first two chapters we give a theoretical background which can be found in more detail in [KS]. See also [Dub, FHST, HT, Lol, Sta] for a review of the classical BRS symmetry.

- The next four chapters give different examples. In the first three the reduced symplectic manifold is $T^{*} S^{n}$ and we quantize linear momentum and observables related to the geodesic flow. In the last example we discuss the spin phase space $S^{2}$. Especially we (re)consider a problem encountered in the geometric quantization of the spin-spin interaction (fine structure) Hamiltonian [DET].

- In the last chapter we discuss the results and make some final remarks.

\section{The Classical BRS Symmetry}

Let $(M, \omega)$ be a symplectic manifold, $G$ a connected Lie group with Lie algebra $\mathscr{G}$ and let $\varphi: G \rightarrow \operatorname{Diff}(M)$ be a symplectic action of $G$ admitting a momentum mapping [Sou1] $\Psi: M \rightarrow \mathscr{G}^{*}\left(\right.$ or $J: \mathscr{G} \rightarrow C^{\infty}(M)$ with $J(\xi)(x) \equiv \Psi(x)(\xi)$ ). We assume that $\Psi$ is Ad-equivariant, or equivalently that $J$ is a Lie algebra morphism from $\mathscr{G}$ to the Poisson algebra $\left(C^{\infty}(M),\{\},\right)$. We furthermore assume that the origin $0 \in \mathscr{G}^{*}$ is a weakly regular value of $\Psi$, in particular that the constraint set $C=\Psi^{-1}(0)$ is a (closed) submanifold of $M$. Finally we assume that the quotient $B=C / G$ is a manifold for which the canonical projection $\pi: C \rightarrow B$ is smooth. We then can apply the Marsden-Weinstein reduction theorem [MW] to affirm that $B$ carries a unique symplectic structure $\omega_{B}$ such that $\left.\omega\right|_{C}=\pi^{*} \omega_{B}$.

What we are interested in is the relation between the Poisson algebras $C^{\infty}(M)$ and $C^{\infty}(B)$. Define $I$ as the ideal (with respect to the pointwise multiplicative structure) of $C^{\infty}(M)$ generated by the functions $J(\xi)$ with $\xi \in \mathscr{G}$, i.e. $I=J(\mathscr{G}) . C^{\infty}(M)$. Since $0 \in \mathscr{G}^{*}$ is (supposed to be) a weakly regular value of $\Psi, I$ can be identified with those functions on $M$ which vanish on $C$. By definition of $B$ as $C / G, C^{\infty}(B)$ is the space of functions on $C$ which are invariant under the $G$-action. Using that $G$ is connected and $J$ a morphism, we then can make the following identifications:

$$
\begin{gathered}
C^{\infty}(C) \cong C^{\infty}(M) / I, \\
C^{\infty}(B) \cong\left\{f \in C^{\infty}(M) \mid \forall \xi \in \mathscr{G}:\{J(\xi), f\} \in I\right\} / I .
\end{gathered}
$$

Again, because $J$ is a morphism, the Poisson bracket (from now on abbreviated as $\mathrm{PB}$ ) in $C^{\infty}(M)$ induces a bracket in the right-hand side of (1.2) which coincides with the PB in $C^{\infty}(B)$ under the identification (1.2). In particular, let $f \in C^{\infty}(M)$ be a representative of $f_{B} \in C^{\infty}(B)$ according to (1.2), then the Hamiltonian vector field of $f$ on $(M, \omega)$ is tangent to $C$ and its restriction to $C$ projects down to the Hamiltonian vector field of $f_{B}$ on $\left(B, \omega_{B}\right)$.

We can summarize this discussion by saying that, under some regularity conditions $\left(0 \in \mathscr{G}^{*}\right.$ weakly regular value, $\pi$ smooth) and the assumptions that $J$ be a morphism and $G$ connected, we can solve a dynamical equation $\dot{x}_{B}=\left\{H_{B}, x_{B}\right\}$ on $B$ with initial conditions $x_{B}(0)=y_{B}$ just by choosing representatives $H$ and $y$ in 
$C^{\infty}(M)$ for $H_{B}$ and $y_{B}$ according to (1.2) and then solving the dynamical equation $\dot{x}=\{H, x\}$ on $M$ with initial conditions $x(0)=y$. One might hope to find representatives for which the corresponding equation on $M$ is easier to solve than the original one on $B$.

We now proceed, following closely [KS], to translate the relation (1.2) between $C^{\infty}(M)$ and $C^{\infty}(B)$ in (co)homological terms. First of all, we consider the vector space $K=\Lambda \mathscr{G} \otimes C^{\infty}(M)$ with the multiplication $(\xi \otimes f) .(\eta \otimes g)=\xi \wedge \eta \otimes f . g$ and the grading induced by the natural grading of the exterior algebra $\Lambda \mathscr{G}$. This turns $K$, which is called the Koszul complex, into a graded super-commutative super algebra. We then consider the boundary operator $\delta: K \rightarrow K$ defined by

$$
\begin{gathered}
\delta(1 \otimes f)=0, \quad \delta(\xi \otimes f)=1 \otimes f . J(\xi) \quad\left(f \in C^{\infty}(M) ; \xi \in \mathscr{G}\right), \\
\delta\left(k . k^{\prime}\right)=\delta(k) \cdot k^{\prime}+(-1)^{\operatorname{deg}(k)} k . \delta\left(k^{\prime}\right) \quad\left(k, k^{\prime} \in K\right) .
\end{gathered}
$$

In this way, $\delta$ is a super-derivation of degree -1 and one easily verifies that $\delta^{2}=0$. Hence we can define the homology groups $H_{q}(K)=\operatorname{ker}(\delta)^{q} / \operatorname{im}(\delta)^{q}$ and especially

$$
H_{0}(K)=\left(1 \otimes C^{\infty}(M)\right) /\left(1 \otimes J(\mathscr{G}) . C^{\infty}(M)\right) \cong C^{\infty}(M) / I \cong C^{\infty}(C) .
$$

In order to obtain $C^{\infty}(B)$ in a cohomological way, we need some basic definitions. Let $E$ be a $\mathscr{G}$-module, i.e. a vector space with a Lie algebra morphism $\rho: \mathscr{G} \rightarrow \operatorname{End}(E)$. The cohomology $H^{*}(\mathscr{G}, E)$ of $\mathscr{G}$ with values in $E$ is then defined as follows. Consider the vector space $L=\Lambda \mathscr{G}^{*} \otimes E$ with the natural grading of $\Lambda \mathscr{G}^{*}$ and choose a basis $\left\{\xi_{1}, \xi_{2}, \ldots, \xi_{n}\right\}$ for $\mathscr{G}$ with its dual basis $\left\{\alpha^{1}, \alpha^{2}, \ldots, \alpha^{n}\right\}$ for $\mathscr{G}^{*}$. Define the coboundary operator $d: L \rightarrow L$ by

$$
d(\mu \otimes k)=d \mu \otimes k+(-1)^{\operatorname{deg}(\mu)} \sum_{i} \mu \wedge \alpha^{i} \otimes \rho\left(\xi_{i}\right) k,
$$

where $d \mu$ is the exterior derivative of $\mu \in \Lambda \mathscr{G}^{*}$ (seen as an invariant form on the group $G$ ), or defined in an algebraic way by

$$
\begin{gathered}
d \alpha^{i}=-\frac{1}{2} \sum_{j, k} C_{j k}^{i} \alpha^{j} \wedge \alpha^{k}, \quad d l=0, \\
d\left(\mu \wedge \mu^{\prime}\right)=d \mu \wedge \mu^{\prime}+(-1)^{\operatorname{deg}(\mu)} \mu \wedge d \mu^{\prime}
\end{gathered}
$$

with $\left[\xi_{j}, \xi_{k}\right]=\sum_{i} C_{j k}^{i} \xi_{i}$. It is elementary to verify that $d^{2}=0$ and that $d$ raises the degree by 1 . Hence we can consider the cohomology groups $H^{q}(\mathscr{G}, E)=\operatorname{ker}(d)^{q} / \operatorname{im}(d)^{q}$ and in particular

$$
H^{0}(\mathscr{G}, E)=\operatorname{ker}(d)^{0}=\bigcap_{\xi \in \mathscr{G}} \operatorname{ker}(\rho(\xi))=\mathscr{G} \text {-invariants of } E .
$$

To apply this construction to $K$ and $H_{0}(K)$, they have to be $\mathscr{G}$-modules. We define the representation $\rho$ of $\mathscr{G}$ on $K$ by

$$
\begin{gathered}
\rho(\xi)(1 \otimes f)=1 \otimes\{J(\xi), f\}, \\
\rho(\xi)(\eta \otimes f)=[\xi, \eta] \otimes f+\eta \otimes\{J(\xi), f\} \quad\left(f \in C^{\infty}(M) ; \xi, \eta \in \mathscr{G}\right), \\
\rho(\xi)\left(k . k^{\prime}\right)=(\rho(\xi) k) . k^{\prime}+k . \rho(\xi) k^{\prime} \quad\left(k, k^{\prime} \in K\right) .
\end{gathered}
$$


In this way $K$ becomes a $\mathscr{G}$-module, and since $J$ is a morphism, the action $\rho(\xi)$ commutes with $\delta$, hence $H_{0}(K)$ inherits in a natural way a $\mathscr{G}$-module structure. In particular

$$
\begin{aligned}
H^{0}\left(\mathscr{G}, H_{0}(K)\right) & =\mathscr{G} \text {-invariants of } H_{0}(K) \\
& \cong \mathscr{G} \text {-invariants of } C^{\infty}(C) \cong C^{\infty}(B) .
\end{aligned}
$$

Now the theory of spectral sequences tells us that under certain circumstances one can obtain $H^{0}\left(\mathscr{G}, H_{0}(K)\right)$ as a single cohomology space from the complex $L=\Lambda \mathscr{G}^{*} \otimes K=\Lambda \mathscr{G}^{*} \otimes \Lambda \mathscr{G} \otimes C^{\infty}(M)=\sum_{p, q} \Lambda^{p} \mathscr{G}^{*} \otimes \Lambda^{q} \mathscr{G} \otimes C^{\infty}(M)$. On this complex with the double grading $(p, q)$ the two operators $d$ and $1 \otimes \delta$ commute because $\delta$ commutes with all $\rho(\xi)$. Then define the operator $D$ on $L$ by ${ }^{1} D=d+2(-1)^{p}(1 \otimes \delta)$, i.e.

$$
\begin{gathered}
A \in \Lambda^{p} \mathscr{G}^{*} \otimes \Lambda^{q} \mathscr{G} \otimes C^{\infty}(M) \\
\downarrow \\
D A=d A+2(-1)^{p}(1 \otimes \delta) A \in \Lambda^{p+1} \mathscr{G}^{*} \otimes \Lambda^{q} \mathscr{G} \otimes C^{\infty}(M) \oplus \Lambda^{p} \mathscr{G}^{*} \otimes \Lambda^{q-1} \mathscr{G} \otimes C^{\infty}(M) .
\end{gathered}
$$

If we reshuffle the double grading $(p, q)$ of $L$ to

$$
L=\sum_{t} L^{t}=\sum_{t} \sum_{p=q=t} \Lambda^{p} \mathscr{G}^{*} \otimes \Lambda^{q} \mathscr{G} \otimes C^{\infty}(M),
$$

then $D$ raises the "total degree" $t$ (ghost number) by 1 and moreover, it is easy to show that $D^{2}=0$. With respect to this total degree one then has the cohomology groups $H_{D}^{t}(L)=\operatorname{ker}(D)^{t} / \mathrm{im}(D)^{t}$ and the proof of the following proposition concerning the relation between the (co)homology of $d, \delta$ and $D$ is an elementary exercise in diagram chasing.

(1.15) Proposition. If $H_{q}(K)=0$ for all $q>0$ then

$$
C^{\infty}(B) \cong H^{0}\left(\mathscr{G}, H_{0}(K)\right) \cong H_{D}^{0}(L)
$$

the identification $H_{D}^{0}(L) \rightarrow C^{\infty}(B)$ being given by $[A] \rightarrow\left[A^{(0,0)}\right]$, where $A \in \operatorname{ker}(D)^{0}$ is a representative of the class $[A] \in H_{D}^{0}(L), A^{(0,0)}$ its component in $\Lambda^{0} \mathscr{G}^{*} \otimes \Lambda^{0} \mathscr{G} \otimes C^{\infty}(M) \cong$ $1 \otimes 1 \otimes C^{\infty}(M)$ and $\left[A^{(0,0)}\right]$ the element in $C^{\infty}(B)$ according to formula (1.2).

Remark. This proposition gives us $C^{\infty}(B)$ as a single cohomology group of the complex $L$. It should be noted that if $0 \in \mathscr{G}^{*}$ is a regular value, not only a weakly regular value (i.e. $d J\left(\xi_{1}\right) \wedge \cdots \wedge d J\left(\xi_{n}\right) \neq 0$ on $C \subset M$, where $\left\{\xi_{1}, \ldots, \xi_{n}\right\}$ is a basis of $\mathscr{G}$ ), then a partition of unity argument together with some algebraic considerations show that the condition of the proposition is satisfied. Hence in the regular case the identification $C^{\infty}(B) \cong H_{D}^{0}(L)$ is valid. We refer the interested reader to [Dub, FHST, HT, Sta] to see what can be done if the condition of the proposition is not satisfied.

\footnotetext{
${ }^{1}$ The factor 2 in the definition of $D$ is purely conventional and has no particular significance for the present discussion
} 
The next step in the KS program is to equip $L=\Lambda \mathscr{G}^{*} \otimes \Lambda \mathscr{G} \otimes C^{\infty}(M)$ with the structure of a (super) Poisson algebra in which $D$ and the "total degree" can be represented as taking PB's with elements $\Theta, T d \in L$. First of all one identifies $\Lambda^{*} \otimes \Lambda \mathscr{G}$ with $\Lambda\left(\mathscr{G}^{*} \oplus \mathscr{G}\right)$ as vector spaces in the most natural way: $\alpha \otimes \xi \equiv \alpha \wedge \xi$, together with the canonical injections $\mathscr{G}^{(*)} \rightarrow a=\mathscr{G}^{*} \oplus \mathscr{G}$. Next we can turn $\Lambda a$ with its natural grading in a super Poisson algebra as follows. Its super commutative product is the wedge product in $\Lambda a$ and the super $\mathrm{PB}$ is defined on generators by

$$
\begin{gathered}
\{1,1\}=\left\{1, \alpha^{i}\right\}=\left\{1, \xi_{i}\right\}=\left\{\alpha^{i}, \alpha^{j}\right\}=\left\{\xi_{i}, \xi_{j}\right\}=0, \\
\left\{\alpha^{i}, \xi_{j}\right\}=2 \delta^{i}{ }_{j},
\end{gathered}
$$

where $\left\{\alpha^{1}, \ldots, \alpha^{n}, \xi_{1}, \ldots, \xi_{n}\right\}$ is a basis of $a=\mathscr{G}^{*} \oplus \mathscr{G}$, and by the fact that it is compatible with the super commutative product structure, i.e.

$$
\begin{gathered}
\{A, B\}=(-1)^{1+\operatorname{deg}(A) \cdot \operatorname{deg}(B)}\{B, A\}, \\
\left\{A,\left\{B, B^{\prime}\right\}\right\}=\left\{\{A, B\}, B^{\prime}\right\}+(-1)^{\operatorname{deg}(A) \cdot \operatorname{deg}(B)}\left\{B,\left\{A, B^{\prime}\right\}\right\} \\
\left\{A, B \wedge B^{\prime}\right\}=\{A, B\} \wedge B^{\prime}+(-1)^{\operatorname{deg}(A) \cdot \operatorname{deg}(B)} B \wedge\left\{A, B^{\prime}\right\} .
\end{gathered}
$$

Having defined a super PB on $\Lambda a$, one can define a super Poisson bracket on $L$ by

$$
\{A \otimes f, B \otimes g\}=\{A, B\} \otimes f . g+A \wedge B \otimes\{f, g\},
$$

where $\{f, g\}$ is the $\mathrm{PB}$ in $C^{\infty}(M)$. This, together with the "usual" product $(A \otimes f) .(B \otimes g)=A \wedge B \otimes f . g$, makes $L$ a super Poisson algebra. Finally we define the elements $\Theta, T d \in L$ by

$$
\begin{gathered}
\Theta=-\frac{1}{4} \sum_{i, j, k} C^{i}{ }_{j k} \alpha^{j} \wedge \alpha^{k} \wedge \xi_{i} \otimes 1+\sum_{i} \alpha^{i} \otimes J\left(\xi_{i}\right), \\
T d=\frac{1}{2} \sum_{i} \alpha^{i} \wedge \xi_{i} \otimes 1,
\end{gathered}
$$

where the $C^{i}{ }_{j k}$ 's are the structure constants of $\mathscr{G}$ relative to the basis $\left\{\xi_{i}\right\}$ and where 1 represents the constant function: 1 on $M$. Using the adjoint representation of $L$ on itself given by ad $(a)(b)=\{a, b\}$, we can state the following theorem whose proof can be found in $[\mathrm{KS}]$.

\section{Theorem.}

i) $\operatorname{Center}(L) \cong 1 \otimes \mathbf{R} \subset \Lambda^{0} a \otimes C^{\infty}(M)$,

ii) $\operatorname{ad}(\Theta)=D$,

iii) $a \in L$ has total degree $t$ iff $\operatorname{ad}(T d)(a)=t a$.

\section{Corollary 1.}

$$
\operatorname{ad}(\Theta)^{2}=0 \text { and }\{\Theta, \Theta\}=0
$$

\section{Corollary 2.}

$$
H_{D}^{0}(L)=(\operatorname{ker} \operatorname{ad}(T d) \cap \operatorname{ker} \operatorname{ad}(\Theta)) /(\operatorname{ker} \operatorname{ad}(T d) \cap \operatorname{im} \operatorname{ad}(\Theta))
$$


and $H_{D}^{0}(L)$ inherits a (super) PB from L. Moreover, if the conditions of the proposition are satisfied, then this induced $\mathrm{PB}$ coincides with the $\mathrm{PB}$ of $C^{\infty}(B)$.

Corollary 2 motivates in retrospect this whole reformulation of formula (1.2) which gives a relation between $C^{\infty}(M), \mathscr{G}$ and $C^{\infty}(B)$. In fact, this corollary tells us that all information of the Poisson algebra $C^{\infty}(B)$ is contained in the super Poisson algebra $L$ and that their relation can be expressed purely in mechanical terms. More precisely, $C^{\infty}(B)$ is the subalgebra of $L$ consisting of those Hamiltonians $H$ for which $\Theta$ and $T d$ are conserved quantities, i.e. $\{\Theta, H\}=\{T d, H\}=0$, all modulo trivial elements of the form $\{\Theta, L\}$.

Remark. In the beginning we assumed that $\Psi$ is Ad-equivariant, although this is not always needed. Associated to a general momentum mapping $\Psi$ is an affine action of $G$ on $\mathscr{G}^{*}$ [Sou1]. If we denote by $\mathscr{G}_{0}$ the Lie algebra of the stabilizer subgroup $G_{0}$ of $0 \in \mathscr{G}^{*}$ for this action, then by replacing $G$ (respectively $\mathscr{G}$ )-invariance by $G_{0}$ (respectively $\mathscr{G}_{0}$ )-invariance, the cohomological construction remains valid, in particular $C^{\infty}(B)=\left\{f \in C^{\infty}(M) \mid \forall \xi \in \mathscr{G}_{0}:\{J(\xi), f\} \in I\right\} / I \cong H^{0}\left(\mathscr{G}_{0}, H_{0}(K)\right) \cong$ $H_{D}^{0}\left(\Lambda \mathscr{G}_{0}^{*} \otimes \Lambda \mathscr{G} \otimes C^{\infty}(M)\right)$. However, as soon as one wants to introduce Poisson brackets the construction breaks down if $\mathscr{G}_{0} \neq \mathscr{G}$. Depending upon the context, the condition $\mathscr{G}_{0}=\mathscr{G}$ is formulated as " $\Psi$ is ad-equivariant," "C= $\Psi^{-1}(0)$ is coisotropic" or "the constraints $J(\xi)$ are all first class."

Remark. If $\Psi$ is any momentum mapping and $\mu \in \mathscr{G}^{*}$ a weakly regular value of $\Psi$, then $\Psi^{\prime}=\Psi-\mu$ is a momentum mapping for which $0 \in \mathscr{G}^{*}$ is weakly regular. If $\Psi$ is Ad-equivariant, then $\Psi^{\prime}$ is Ad-equivariant iff $\mu([\mathscr{G}, \mathscr{G}])=0$.

\section{BRS Quantization}

In this section we consider the quantization of the (super) Poisson algebras $C^{\infty}(B)$ and $L$. Usually one means by quantization of a symplectic manifold $(M, \omega)$ a linear map $\tau_{0}$ from some subspace of the Poisson algebra $C^{\infty}(M)$ to (skew) self-adjoint operators on a Hilbert space $\mathscr{H}_{M}$ which is as far as possible a Lie algebra morphism $^{2} \tau_{0}(\{f, g\})=\left[\tau_{0}(f), \tau_{0}(g)\right]$. We have to say "as far as possible" because the fact that $\tau_{0}$ should be a morphism conflicts with an irreducibility condition one usually imposes. Finally one requires that the function constant 1 on $M$ should be mapped to $(i / \hbar)$ times the identity operator 1 . In our case we have a super Poisson algebra $L$, so it is natural to require that a quantization of $L$ is a linear map $\tau$ from some subspace of $L$ to (skew) self-adjoint operators on some Hilbert space $\mathscr{H}_{L}$ which is as far as possible a super Lie algebra morphism.

The general idea to find a quantization of $C^{\infty}(B)$, i.e. the Poisson algebra of the reduced space, is the following. Assuming that the condition of Proposition $(1.15)$ is satisfied, $C^{\infty}(B)$ is given by formulas (1.16) and (1.27) with the induced $\mathrm{PB}$, and one defines the quantization of the reduced problem by a quantized

\footnotetext{
${ }^{2}$ This approach differs by a factor $\not h / i$ from the usual approach in order to obtain a normal Lie algebra morphism
} 
version of this formula

$$
\begin{gathered}
\mathscr{H}_{\text {red }}=(\operatorname{ker} \tau(T d) \cap \operatorname{ker} \tau(\Theta)) /(\operatorname{ker} \tau(T d) \cap \operatorname{im} \tau(\Theta)), \\
\tau_{\text {red }}([H])=\text { the operator on } \mathscr{H}_{\text {red }} \text { induced by } \tau(H),
\end{gathered}
$$

where $H \in L$ is any representative of the class $[H] \in H_{D}^{0}(L)$ representing an observable $H_{B} \in C^{\infty}(B)$ according to formulas (1.16) and (1.27). If $\tau$ is a super Lie algebra morphism for the observables involved, then it is not hard to show the following:

$$
\tau(\Theta)^{2}=0
$$

$(\{\Theta, \Theta\}=0$ and on odd-degree elements use the anti-commutator)

$$
\begin{aligned}
& \{\Theta, H\}=\{T d, H\}=0 \Rightarrow \tau(H)(\operatorname{ker} \tau(T d) \cap \operatorname{im} \tau(\Theta)) \subset \operatorname{ker} \tau(T d) \cap \operatorname{im} \tau(\Theta), \\
& H=\{\Theta, f\} \&\{T d, H\}=0 \Rightarrow \tau(H)(\operatorname{ker} \tau(T d) \cap \operatorname{ker} \tau(\Theta)) \subset \operatorname{ker} \tau(T d) \cap \operatorname{im} \tau(\Theta),
\end{aligned}
$$

and these facts prove that $\left(\mathscr{H}_{\text {red }}, \tau_{\text {red }}\right)$ is a well defined quantization of $C^{\infty}(B)$-modulo the fact that $\tau$ should be a morphism.

We now give the KS approach to the quantization of $L$, while we will point out in due course the remaining open problems. The vector space $a=\mathscr{G}^{*} \oplus \mathscr{G}$ has a natural symmetric bilinear form $\langle$,$\rangle defined by$

$$
\langle\alpha, \xi\rangle=\langle\xi, \alpha\rangle=\alpha(\xi),\left\langle\alpha, \alpha^{\prime}\right\rangle=\left\langle\xi, \xi^{\prime}\right\rangle=0 \quad\left(\alpha, \alpha^{\prime} \in \mathscr{G}^{*} ; \xi, \xi^{\prime} \in \mathscr{G}\right) .
$$

With this non-degenerate bilinear form one can construct the Clifford algebra $C(a)$ and there is an isomorphism of vector spaces $\psi: C(a) \rightarrow \Lambda a$ defined by ${ }^{3}$

$$
\psi^{-1}\left(a_{1} \wedge a_{2} \wedge \cdots \wedge a_{k}\right)=\widehat{a[1} \cdot a_{2} \cdots \cdots a_{k} \hat{]} \quad\left(a_{j} \in a\right) .
$$

If we equip $C(a)$ with the super commutator, it becomes a super Poisson algebra and, moreover, $\psi$ becomes to some extent a super Poisson algebra morphism. More precisely

$$
\forall a, b \in \Lambda a: \min (\operatorname{deg}(a), \operatorname{deg}(b)) \leqq 2 \Rightarrow \psi^{-1}(\{a, b\})=\left[\psi^{-1}(a), \psi^{-1}(b)\right]_{\text {super }}
$$

Inside $C(a)$ one can consider the ideal $N$ generated by $\mathscr{G}$, i.e. $N=C(a) \cdot \mathscr{G}$. The quotient $C(a) / N$ can be canonically identified with $\Lambda \mathscr{G}^{*}$, which then becomes a $C(a)$-module. In this identification, the action of $C(a)$ on $\Lambda^{*}$ is defined by

$$
\alpha(A)=\alpha \wedge A, \quad \xi(A)=2 i(\xi) A, \quad\left(\alpha \in \mathscr{G}^{*}, \xi \in \mathscr{G}, A \in \Lambda^{*}\right),
$$

where $i(\xi) A$ denotes the contraction of the form $A$ with the vector $\xi$.

The idea given in [KS] is that one should quantize $L$ as operators on $\mathscr{H}_{L}=\left(\Lambda g^{*}\right)^{\mathbf{C}} \otimes \mathscr{H}_{M}$, where $\tau_{0}: C^{\infty}(M) \rightarrow \operatorname{End}\left(\mathscr{H}_{M}\right)$ is an "ordinary" quantization of $C^{\infty}(M)$. In fact, with the above construction, we have $\psi^{-1}: \Lambda a \rightarrow \operatorname{End}\left(\left(\Lambda^{\mathscr{G}^{*}}\right)^{\mathrm{C}}\right) \subset$ End $\left(\mathscr{H}_{L}\right)$ and in the same way $\tau_{0}: C^{\infty}(M) \rightarrow \operatorname{End}\left(\mathscr{H}_{L}\right)$. Unfortunately there is in general no hope that the combination $\psi^{-1} \otimes \tau_{0}: L \rightarrow \operatorname{End}\left(\mathscr{H}_{L}\right)$ is a quantization, due to the fact that the PB on $L$ depends upon (super) commutative structures

3 The square brackets denote skew-symmetrization 
(see formula (1.22)!), a structure which is present neither in End $\left(\left(\Lambda^{\mathscr{G}^{*}}\right)^{\mathbf{C}}\right)$ nor in End $\left(\mathscr{H}_{M}\right)$.

In order to make $\mathscr{H}_{L}$ a Hilbert space for a quantization of $L$, we need an inner product on $\left(\Lambda_{\mathscr{G}^{*}}\right)^{\mathbf{C}}$. In [KS, Sect. 11] it is shown that there exists a unique (up to a real factor) non-singular sesquilinear form $\langle$,$\rangle on \left(\Lambda \mathscr{G}^{*}\right)^{\mathrm{C}}$ with the property that the adjoint of the operator $a_{1} \cdot a_{2} \cdots \cdot a_{k} \in C(a)$ on $\left(\Lambda \mathscr{G}^{*}\right)^{\mathbf{C}}$ (with $a_{j} \in a$ ) is given by $a_{k} \cdot a_{k-1} \cdots \cdots a_{1}$. In the finite dimensional case it is easy to give an explicit formula for such a form. One starts by choosing a non-zero element vol $\in \Lambda^{n} \mathscr{G}^{*} \subset\left(\Lambda^{n} \mathscr{G}^{*}\right)^{\mathrm{C}}$, where $n=\operatorname{dim}(\mathscr{G})$ and one defines an involution ' $f$ ' on $\left(\Lambda^{*}\right)^{*}$ ' by

$$
\left(\lambda \alpha^{1} \wedge \alpha^{2} \wedge \cdots \wedge \alpha^{k}\right)^{\dagger}=\bar{\lambda} \alpha^{k} \wedge \alpha^{k-1} \wedge \cdots \wedge \alpha^{1} \quad\left(\lambda \in \mathbf{C}, \alpha^{j} \in \mathscr{G}^{*}\right) .
$$

With this definition one defines the sesquilinear form $\langle$,$\rangle by$

$$
\langle A, B\rangle=i^{n(n-1) / 2} \frac{\pi_{n}\left(A^{\dagger} \wedge B\right)}{\operatorname{vol}} \quad\left(A, B \in\left(\Lambda \mathscr{G}^{*}\right)^{\mathbf{C}}\right),
$$

where $\pi_{n}\left(A^{\dagger} \wedge B\right)$ is the component of $A^{\dagger} \wedge B$ in $\left(\Lambda^{n} \mathscr{G} *\right)^{\mathbf{C}}$.

With these preparations, the elements $\Theta$ and $T d$ and only these are quantized in $[\mathrm{KS}]$ by the following operators:

and

$$
\begin{aligned}
\tau(\Theta) & =\left(-\frac{1}{4} \sum_{i, j, k} C_{j k}^{i} \alpha^{j} \alpha^{k} \xi_{i}-\frac{1}{2} \sum_{j, k} C_{j k}^{j} \alpha^{k}\right) \otimes 1+\sum_{i} \alpha^{i} \otimes \tau_{0}\left(J\left(\xi_{i}\right)\right) \\
& =\psi^{-1}\left(-\frac{1}{4} \sum_{i, j, k} C^{i}{ }_{j k} \alpha^{j} \wedge \alpha^{k} \wedge \xi_{i}\right) \otimes 1+\left(\psi^{-1} \otimes \tau_{0}\right)\left(\sum_{i} \alpha^{i} \otimes J\left(\xi_{i}\right)\right)
\end{aligned}
$$

$$
\tau(T d)=\frac{1}{2} \sum_{i} \alpha^{i} \xi_{i}=\left(\psi^{-1}\left(\frac{1}{2} \sum_{i} \alpha^{i} \wedge \xi_{i}\right)+\frac{n}{2} 1\right) \otimes 1 .
$$

If $\tau_{0}: C^{\infty}(M) \rightarrow \operatorname{End}\left(\mathscr{H}_{M}\right)$ is a morphism on the $J\left(\xi_{i}\right)$ 's, i.e. $\left[\tau_{0}\left(J\left(\xi_{j}\right)\right), \tau_{0}\left(J\left(\xi_{k}\right)\right)\right]=$ $\tau_{0}\left(\left\{J\left(\xi_{j}\right), J\left(\xi_{k}\right)\right\}\right)=\sum_{i} C_{j k}^{i} \tau_{0}\left(J\left(\xi_{i}\right)\right)$, then this $\tau(\Theta)$ has the following properties:

i) $\tau(\Theta)$ is skew self-adjoint,

ii) $\tau(\Theta)^{2}=0$,

iii) $[\tau(\Theta), \tau(T d)]=\tau(\Theta)$.

Since the eigenspaces of $\tau(T d)$ are exactly the spaces $\left(\Lambda^{k} \mathscr{G}^{*}\right)^{\mathbf{C}} \otimes \mathscr{H}_{M}, \operatorname{ker} \tau(T d)$ is isomorphic (as vector space) to $\mathscr{H}_{M}$ and formula (2.1) gives

$$
\begin{aligned}
\mathscr{H}_{\text {red }} & \cong\left\{f \in \mathscr{H}_{M} \mid \forall i=1, \ldots, n: \tau_{0}\left(J\left(\xi_{i}\right)\right) f=\frac{1}{2} \sum_{j} C^{j}{ }_{j i} f\right\} \\
& \cong\left\{f \in \mathscr{H}_{M} \mid \forall \xi \in \mathscr{G}: \tau_{0}(J(\xi)) f=-\frac{1}{2} \operatorname{Tr}(\operatorname{ad}(\xi)) f\right\} .
\end{aligned}
$$

This seems a very nice result, but some questions must be raised.

-First, $\tau(T d)$ is not skew self-adjoint, so $\tau(T d)$ does not fit our general ideas on quantization. One might notice that $\psi^{-1}\left(\sum_{i} \alpha^{i} \wedge \xi_{i}\right) \otimes 1$ is skew self-adjoint, but using this operator for $\tau(T d)$ poses another problem: its spectrum is 
$\{-(n / 2), \ldots,(n / 2)\}$ which does not contain 0 for odd $n$; this would imply that $\mathscr{H}_{\text {red }}=\{0\}$ for odd-dimensional $\mathscr{G}$.

- Second, the $\tau_{0}\left(J\left(\xi_{i}\right)\right.$ )'s are skew self-adjoint (otherwise $\tau(\Theta)^{2} \neq 0$ ) and the structure constants are real, so $\mathscr{H}_{\text {red }}=\{0\}$ if $\sum_{j} C_{j i}^{j} \neq 0$ for some $i$. See however example 3.

- Third, the natural scalar product on $\mathscr{H}_{\text {red }}$ induced by the scalar product of $\mathscr{H}_{L}$ is identically zero (this explains why the skew self-adjoint operator $\psi^{-1}\left(\sum_{i} \alpha^{i} \wedge \xi_{i}\right) \otimes 1$ can have real eigenvalues $)$ and hence does not coincide with the inner product induced by $\mathscr{H}_{M}$.

Apart from these questions, we do not have a general quantization $\tau$ for $L$, so how should we quantize $H \in \operatorname{ker} \operatorname{ad}(T d) \cap \operatorname{ker} \operatorname{ad}(\Theta)$, a result we need in our formula (2.2) for $\tau_{\mathrm{red}}([H])$ ? If we assume that there exists a function $H_{0} \in C^{\infty}(M)$ which is globally invariant under the $G$-action and which induces $[H] \approx H_{B} \in C^{\infty}(B)$, then $\left[1 \otimes H_{0}\right]=[H]$ in $H_{D}^{0}(L)$. One then might "guess" that $\tau\left(1 \otimes H_{0}\right)=1 \otimes \tau_{0}\left(H_{0}\right)$. However, since we do not have a general $\tau$, it remains to show that this $\tau\left(1 \otimes H_{0}\right)$ induces a well-defined operator on $\mathscr{H}_{\text {red }}$. A sufficient condition for this to be the case is that $\tau_{0}\left(H_{0}\right)$ commutes with $\tau_{0}(J(\mathscr{G}))$.

This finishes our discussion of the [KS] approach to BRS-quantization. To summarize, modulo some natural assumptions which are not motivated by this theory, the quantization of the constrained system $C^{\infty}(B)$ via a quantization of $C^{\infty}(M)$ is given by the following

\section{BRS-KS Quantization Procedure.}

1. Quantize the constrained functions $J(\xi)$ by $\tau_{0}(J(\xi))$ on $\mathscr{H}_{M}$ and hope that $\tau_{0}$ is a morphism on the $\xi$ 's in $\mathscr{G}$.

2. Define $\mathscr{H}_{\text {red }}=\left\{f \in \mathscr{H}_{M} \mid \forall \xi \in \mathscr{G}: \tau_{0}(J(\xi)) f=-\frac{1}{2} \operatorname{Tr}(\operatorname{ad}(\xi)) f\right\}$ with an inner product induced by $\mathscr{H}_{M}$ (if it exists).

3. To quantize $H_{B} \in C^{\infty}(B)$, find if possible a globally $G$-invariant representative $H_{0} \in C^{\infty}(M)$; quantize $H_{0}$ by $\tau_{0}\left(H_{0}\right)$ on $\mathscr{H}_{M}$ and hope that $\tau_{0}\left(H_{0}\right)$ commutes with $\tau_{0}(J(\xi))(\forall \xi \in \mathscr{G})$.

4. Define $\tau_{\text {red }}\left(H_{B}\right)=\left.\tau_{0}\left(H_{0}\right)\right|_{\mathscr{H}_{\text {red }}}$.

In the next sections we will see how this procedure works in practice. We will use the geometric quantization procedure to provide the quantization $\tau_{0}$ for $C^{\infty}(M)$ and also to provide a direct quantization $\tau_{B}: C^{\infty}(B) \rightarrow \operatorname{End}\left(\mathscr{H}_{B}\right)$ which can be compared with the BRS-quantization $\left(\mathscr{H}_{\text {red }}, \tau_{\text {red }}\right)$.

\section{The Geodesic Flow and Boosts On Spheres}

Reduction by Dilations. The cotangent bundle $B=T^{*} S^{n}$ with canonical 1-form $\theta_{B}$ can be viewed as a $\mathbf{R}_{+}^{*}$-reduction for the symplectic manifold $(M, \omega)$, where $M=T^{*}\left(\mathbf{R}^{n+1} \backslash\{0\}\right), \omega=d \theta$ and $\theta=\langle P, d Q\rangle$. We denote by $\langle$,$\rangle the standard$ 
Euclidean metric of $\mathbf{R}^{n+1}$ and by $\|\quad\|$ the induced norm. The group $\mathbf{R}_{+}^{*}$ of dilations ${ }^{4}$ acts symplectically on $(M, \omega)$ according to $(P, Q) \rightarrow(P / a, Q a)\left(a \in \mathbf{R}_{+}^{*}\right)$ and the associated momentum mapping $\Psi: M \rightarrow \mathbf{R}$ reads

$$
\Psi(P, Q)=\langle P, Q\rangle .
$$

The $2 n+1$ dimensional submanifold $C=\Psi^{-1}(0)$ is the coisotropic constrained manifold we are interested in. Note that 0 is a regular value of $\Psi$. We have

$$
B=C / \mathbf{R}_{+}^{*} \cong T^{*} S^{n}
$$

and the projection $C \rightarrow B$ is given by $(P, Q) \rightarrow(p, q)=(P\|Q\|, Q /\|Q\|)$ where we have identified $T^{*} S^{n}$ with $\left\{(p, q) \in T^{*} \mathbf{R}^{n+1} \mid\|q\|=1,\langle p, q\rangle=0\right\}$. The MarsdenWeinstein reduction theorem then tells us that $B$ is endowed with a symplectic structure: here, the canonical one since the induced 1 -form $\left.\theta\right|_{C}$ descends to $B$ as $\theta_{B}=\langle p, d q\rangle$.

Geodesic Flow and Boosts. There are two Hamiltonians we would like to quantize as a first test of the method of Kostant and Sternberg, namely $h_{1}=\frac{1}{2}\|p\|^{2}$ (the kinetic energy of a free particle moving on a sphere) and $h_{2}=\langle p, \beta\rangle$ (the projection of the linear momentum in the direction of $\left.\beta \in \mathbf{R}^{n+1}\right)$. These two functions in $C^{\infty}(B)$ can be represented by the following globally $\mathbf{R}_{+}^{*}$-invariant functions in $C^{\infty}(M)$ :

$$
H_{1}=\frac{1}{2}\|P\|^{2}\|Q\|^{2}, \quad H_{2}=\|Q\|\langle P, \beta\rangle .
$$

The geodesic flow on $T^{*} S^{n}$ corresponds to the projection of the Hamiltonian flow of $H_{1}$ restricted to the constrained manifold $C$. See [Mos2] for a discussion of other choices of Hamiltonians. The flow of $H_{1}$ is given explicitly by

$$
\left\{\begin{array}{l}
P_{t}=e^{-t\langle P, Q\rangle}\left\{\cos (\Omega t) P+\frac{\sin (\Omega t)}{\Omega}\left(\langle P, Q\rangle P-\|P\|^{2} Q\right)\right\} \\
Q_{t}=e^{t\langle P, Q\rangle}\left\{\cos (\Omega t) Q+\frac{\sin (\Omega t)}{\Omega}\left(\|Q\|^{2} P-\langle P, Q\rangle Q\right)\right\}
\end{array},\right.
$$

where $\Omega=\sqrt{\|P\|^{2}\|Q\|^{2}-\langle P, Q\rangle^{2}}$. Let us emphasize that $H_{1}$ can be interpreted as the kinetic energy corresponding to the geodesic flow on the configuration space $\mathbf{R}^{n+1} \backslash\{0\}$ endowed with the conformally flat metric $\|Q\|^{-2}\langle$,$\rangle . As for the$ so-called boost-Hamiltonian $h_{2}$, we will show in Sect. 5 that it is indeed related to a Lorentz boost in the orthogonal group $O(n+1,1)$.

Prequantization and Polarizations. We briefly introduce the main ingredients that we need to quantize observables in the framework of geometric quantization [Sou1, Kos, Sni, Woo]. Our symplectic manifold is a cotangent bundle, hence prequantization is trivial: the prequantum bundle over $(M, \omega)$ is the circle-bundle $M \times U(1)$ with connection form $\alpha=\theta+(d z / i z)(z \in U(1))$. We assume $n>1$ (M simply connected) to insure uniqueness of prequantization. In order to undertake the program of geometric quantization, we have to choose a polarization $F$ of our

${ }^{4}$ A subgroup of $\mathrm{Sl}(2, \mathbf{R})$ - see below 
symplectic manifold. The most natural choice at hand is the vertical polarization of contangent bundles: $Q=$ const. Associated to this choice, we have a natural Hilbert space structure given by the square integrable half-densities of the configuration space $M / F=\mathbf{R}^{n+1} \backslash\{0\}$. This Hilbert space $\mathscr{H}_{M}$ can identified with $L^{2}\left(\mathbf{R}^{n+1}, d Q\right)$, where " $d Q$ " is the Lebesgue measure. The inner product of $\mathscr{H}_{M}$ is given by $\langle f, g\rangle=\int \overline{f(Q)} g(Q) d Q$.

Quantum Reduction by Dilations. Since $\mathbf{R}_{+}^{*}$ preserves the polarization $F$, the momentum mapping $\Psi$ can be quantized directly without the Blattner-KostantSternberg (BKS) pairing procedure [Blal, GS]. The operator $\tau(\Psi)$ is thus merely given by the generator of the natural unitary representation of $\mathbf{R}_{+}^{*}$ on $\mathscr{H}_{M}: f^{\#} \rightarrow a^{*} f^{\#}\left(a \in \mathbf{R}_{+}^{*}\right)$, where $f^{\#}=f \otimes \sqrt{|d Q|}$, hence

$$
\tau(\Psi) f(Q)=Q \cdot \frac{\partial f}{\partial Q}+\frac{n+1}{2} f(Q)
$$

The quantum constraint $\tau(\Psi) f=0$ thus implies

$$
f(Q)=\|Q\|^{-(n+1) / 2} \hat{f}\left(\frac{Q}{\|Q\|}\right)
$$

with $\hat{f}$ a function on $S^{n}$. If we now (arbitrarily) endow functions on $S^{n}$ with the standard inner product given by the $S O(n+1)$-invariant measure on $S^{n}$, we are able to identify the reduced Hilbert space $\mathscr{H}_{\text {red }}$ with $L^{2}\left(S^{n}\right)$. Now $L^{2}\left(S^{n}\right)$ is exactly the Hilbert space we would have obtained by quantizing $B=T^{*} S^{n}$ directly using the vertical polarization, i.e. we have found an identification $\mathscr{H}_{B} \cong L^{2}\left(S^{n}\right)$. There is only one problem: if we start with functions $f \in L^{2}\left(\mathbf{R}^{n+1}\right)$ then $\mathscr{H}_{\text {red }}=\{0\}$ because for any non-zero element $\widehat{f} \in L^{2}\left(S^{n}\right)$ the function $f$ given by (3.6) is not in $L^{2}\left(\mathbf{R}^{n+1}\right)$. In order to get the desired result, we have first to enlarge $L^{2}\left(\mathbf{R}^{n+1}\right)$ to all functions (in other examples even to distributions) on $\mathbf{R}^{n+1}$, then to apply the quantum constraint (3.5) and finally restricting to $L^{2}$ functions (but now on a different measure space). Obviously we will do this and we will identify $\mathscr{H}_{\text {red }}$ on the one hand as a subspace of $L^{2}\left(\mathbf{R}^{n+1}\right)$-enlarged and on the other hand with $L^{2}\left(S^{n}\right)$.

BRS-Quantization of $H_{1}$ and $H_{2}$. Unfortunately the Hamiltonian $H_{1}$ does not preserve the polarization $F$ and this leads us to consider the Hilbert space $\mathscr{H}_{t}$ associated with the image $F_{t}$ of the original (vertical) polarization $F$ under the flow of $H_{1}$. The BKS pairing of $\mathscr{H}_{M}=\mathscr{H}_{t=0}$ and $\mathscr{H}_{t}$ is given by

$$
\left\langle g, f_{t}\right\rangle_{\mathscr{H}_{0}, \mathscr{H}_{t}}=(2 \pi)^{-(n+1) / 2} \int_{M} e^{-i t H(P, Q)} \overline{g(Q)} f\left(Q_{t}\right) K(P, Q, t) d P d Q,
$$

where $K(P, Q, t)=e^{t\langle P, Q\rangle(n+1) / 2}\|Q\|^{n+1} t|\sin (\Omega t) / \Omega|^{(n-1) / 2}$ and " $d P d Q$ " is the Liouville measure.

The method of the stationary phase leads to: $\left\langle g, f_{t}\right\rangle_{\mathscr{H}_{0}, \mathscr{H}_{t}}=\left\langle g, f+t \tau\left(H_{1}\right) f\right\rangle_{\mathscr{H}_{M}}$ $+o(t)$ and the quantized anti self-adjoint Hamiltonian reads

$$
\tau\left(H_{1}\right) f(Q)=-\frac{i}{2}\left\{\|Q\|^{2} \Delta+2 Q \cdot \frac{\partial}{\partial Q}-\frac{(n-1)^{2}}{4}-\frac{n(n-1)}{6}\right\} f(Q) .
$$


The Hamiltonian $\mathrm{H}_{2}$ is linear in momentum, it thus leaves the vertical polarization invariant and can be quantized directly and

$$
\tau\left(H_{2}\right) f(Q)=\|Q\| \beta \cdot \frac{\partial f}{\partial Q}+\frac{\langle\beta, Q\rangle}{2\|Q\|} f(Q) .
$$

Now $\tau\left(H_{1}\right)$ and $\tau\left(H_{2}\right)$ commute with the quantum constraint (3.5), so they induce well defined operators on $\mathscr{H}_{\text {red }} \cong L^{2}\left(S^{n}\right)$. Expanding $\Delta_{\mathbf{R}^{n+1}}$ in its radial and spherical parts, we find the induced operators:

$$
\tau_{\text {red }}\left(h_{1}\right) \hat{f}(q)=-\frac{i}{2}\left\{\Delta_{S^{n}}-\frac{R}{6}\right\} \hat{f}(q),
$$

where $R=n(n-1)$ is the scalar curvature of $S^{n}$ and

$$
\tau_{\text {red }}\left(h_{2}\right) \hat{f}(q)=(\beta-q\langle q, \beta\rangle) \cdot \frac{\partial \hat{f}}{\partial q}-\frac{n}{2}\langle q, \beta\rangle \hat{f}(q) .
$$

Discussion. Had we quantized $h_{1}$ and $h_{2}$ by applying the methods of geometric quantization directly on $T^{*} S^{n}$ (using the vertical polarization and the identification $\mathscr{H}_{\text {red }} \cong \mathscr{H}_{B}$ as described above), we would have got exactly the same result.

Note that quantization of $h_{1}$ is nothing but a special case of a general result known as the quantization of the geodesic flow of Riemannian manifolds [Elh, Sni]. In fact the same is true for the calculation to obtain (3.7) if we endow $\mathbf{R}^{n+1} \backslash\{0\}$ with the modified metric $\|Q\|^{-2}\langle$,$\rangle (see above).$

To summarize: with the choice of the vertical polarization for $M$ and $B$, quantization and reduction do commute.

Remarks. We could have chosen a different polarization on $M$, e.g. the anti-holomorphic polarization which realizes $\mathscr{H}_{M}$ as holomorphic functions on $\mathrm{C}^{n+1}$ with a Gaussian measure: the Bargmann representation. Quantizing $H_{1}$ and the constraint $\Psi$ with this polarization and using the standard isomorphism between the Schrödinger and Bargmann representation, we find the same result for $\tau(\Psi)$, but a different one for $\tau\left(H_{1}\right)$. In fact, the operator induced on $\mathscr{H}_{\text {red }} \cong L^{2}\left(S^{n}\right)$ is given by:

$$
\tau_{\mathrm{red}}^{\prime}\left(h_{1}\right) \hat{f}(q)=-\frac{i}{2}\left\{\Delta_{\mathrm{S}^{n}}+\frac{n+1}{2}\right\} \hat{f}(q)
$$

which is different from our previous result.

If we had used Weyl quantization on $T^{*}\left(\mathbf{R}^{n+1} \backslash\{0\}\right)$ instead of geometric quantization, we would have got yet another result

$$
\tau_{\text {red }}^{\prime \prime}\left(h_{1}\right) \hat{f}(q)=-\frac{i}{2}\left\{\Delta_{S^{n}}+\frac{n^{2}-1}{4}\right\} \hat{f}(q) .
$$

\section{The Kepler Manifold or the Null Geodesics of Compactified Minkowski Space-time}

Introduction. We wish to illustrate now the case of a symplectic reduction with $S l(2, \mathbf{R})$. The example we will be dealing with is related to the Kepler manifold 
$\dot{T} S^{3}$ of "regularized" planetary motion ${ }^{5}$ corresponding to the negative energy orbits in the Newtonian gravitational field [Mos1, Sou2].

The manifold we are interested in is the cone $B$ of those $\mu \neq 0$ in the dual of the Lie algebra $o(4,2)$ that are nilpotent: $\mu^{2}=0$ (see [Sou2]). It turns out that $B$ is a co-adjoint orbit of the Lie group $O(4,2)$ that consists of two copies of the Kepler manifold

$$
B=B_{+} \cup B_{-}, \quad B_{ \pm} \cong \dot{T} S^{3} .
$$

The "neutral" component $B_{+}$is endowed with the canonical 1-form and $B_{-}$ (obtained by the symmetry $\mu \rightarrow-\mu$ ) with the opposite 1-form: $\theta_{B \pm}= \pm\langle p, d q\rangle$. We denote by $\langle$,$\rangle the Euclidean scalar product in \mathbf{R}^{4}$. Generalization to $O(n+1,2)$ is straightforward: $B_{ \pm} \cong \dot{T} S^{n}$.

We will construct the symplectic manifold $\left(B, \omega_{B}\right)$ by means of the Marsden-Weinstein reduction with $G=S l(2, \mathbf{R})$ that will make a clear connection with the description of (spinless and) massless particles living in Minkowski space-time, more precisely in its conformal compactification $S^{n, 1}=\left(S^{n} \times S^{1}\right) / \mathbf{Z}_{2}$.

Symplectic Reduction. We start with $\mathbf{R}^{n+3}$ which we endow with the metric $g=\operatorname{diag}(1,1, \ldots, 1,-1,-1)$ and use $\mathbf{R}^{n+1,2}$ as a shorthand for $\left(\mathbf{R}^{n+3}, g\right)$. With this metric we identify $T \mathbf{R}^{n+1,2}$ with $T^{*} \mathbf{R}^{n+1,2}$ and define $M=\left\{(P Q) \in T R^{n+1,2} \mid\right.$ $P \wedge Q \neq 0\}$ endowed with the canonical symplectic form inherited from that of $T^{*} \mathbf{R}^{n+1,2}$. On $M$ we define the 1 -form $\theta=1 / 2(\bar{P} . d Q-d \bar{P} . Q)$, where $\bar{V}=g(V$, denotes the covector associated to the vector $V$ by the metric $g$. Note that $\omega=d \theta$ and that $S l(2, \mathbf{R})$ acts symplectically on $(M, \omega)$ according to

$$
(P Q) \rightarrow(P Q)\left(\begin{array}{ll}
a & b \\
c & d
\end{array}\right) \quad(a d-b c=1) .
$$

This right-action moreover preserves $\theta$ and hence [Sou1] gives rise to a momentum mapping $\Psi: M \rightarrow s l(2, \mathbf{R})^{*}$ that reads

$$
\Psi(P, Q)=\left(\begin{array}{cc}
\bar{P} \cdot Q & \bar{Q} \cdot Q \\
-\bar{P} \cdot P & -\bar{P} \cdot Q
\end{array}\right) .
$$

There exists another obvious group of symplectomorphisms of $(M, \omega)$, namely $O(n+1,2)$ whose left-action reads

$$
(P Q) \rightarrow(A P \quad A Q) \quad(A \in O(n+1,2)) .
$$

The associated momentum mapping $\Phi: M \rightarrow o(n+1,2)^{*}$ is given by

$$
\Phi(P, Q)=P \cdot \bar{Q}-Q \cdot \bar{P} .
$$

Note that in both (4.3) and (4.5) we have identified the Lie algebra with its dual using the scalar product $\langle\xi, \eta\rangle=\frac{1}{2} \operatorname{Tr}(\xi . \eta)$ on the Lie algebra. The actions of $S l(2, \mathbf{R})$ and $O(n+1,2)$ commute (dual pairs) and $\Phi(M)$ is the cone of rank 2 matrices in $o(n+1,2)$. The origin $0 \in s l(2, \mathbf{R})$ is clearly a regular value for $\Psi$.

The constrained manifold to consider next is the $2 n+3$ dimensional smooth

${ }^{5} \dot{T} Q$ is the tangent bundle of $Q$ with the zero section deleted 
submanifold $C=\Psi^{-1}(0)$ defined by the equations [GS],

$$
\bar{P} \cdot P=\bar{Q} \cdot Q=\bar{P} \cdot Q=0
$$

which turns out to have two connected components: $C=C_{+} \cup C_{-}$characterized by

$$
\rho=\bar{P} . T \cdot Q\left\{\begin{array}{lll}
>0 & \text { on } & C_{+} \\
<0 & \text { on } & C_{-}
\end{array}\right.
$$

where $T=\left(\begin{array}{ccc}0 & 0 & 0 \\ 0 & 0 & -1 \\ 0 & 1 & 0\end{array}\right)$ is a $S O(2)$-generator in $o(n+1,2)$. The reduced symplectic manifold

$$
B=C / \operatorname{Sl}(2, \mathbf{R})
$$

is nothing but the co-adjoint orbit of $O(n+1,2)$,

$$
B=\Phi\left(\Psi^{-1}(0)\right) \text {. }
$$

We find $B=B_{+} \cup B_{-}$with $B_{ \pm}=\Phi\left(C_{ \pm}\right) \cong \dot{T} S^{n}$. Moreover the 1-form $\left.\theta\right|_{C}$ passes to the quotient as the 1 -form $\theta_{B}$ given above.

Remarks. In the case $n=3,\left(B_{+}, \omega_{+}\right)$is symplectomorphic to the space of regularized bounded Keplerian motions [Sou2]. The fact that $O(4,2)$ is indeed a group of symplectomorphisms is the geometrical manifestation of an accidental symmetry of the model. The famous $O(4)$ sub-symmetry was originally discovered by Pauli in a quantum mechanical context; it is responsible for the high degeneracy of the spectrum of the hydrogen atom.

- The orbit $(B, \omega)$ is symplectomorphic to the space of motions of a massless (and spinless) particle in the conformally compactified Minkowski space-time $S^{n, 1}$. To justify our claim, we recall the fibration $C \rightarrow S^{n, 1}:(P Q) \rightarrow Q \bmod \mathbf{R}^{*}$ which factors through the null tangent bundle $C \rightarrow T_{0} S^{n, 1} \rightarrow S^{n, 1}$ because $T_{0} S^{n, 1} \cong C / S$, where $S$ is the 2-dimensional solvable subgroup of lower triangular matrices in $G=S l(2, \mathbf{R})$. Our orbit $B=C / G$ is clearly symplectomorphic to $T_{0} S^{n, 1} / \mathbf{P}_{1}(\mathbf{R})$, that is to the space of null geodesics of $S^{n, 1}$, i.e. the space of motions of a massless scalar particle dwelling in the conformally compactified Minkowski space-time. Here time is compact: $\mathbf{P}_{1}(\mathbf{R}) \cong G / S$ and the two components $B_{ \pm}$correspond to the positive $(\rho>0)$ respectively negative $(\rho<0)$ energy classical states of that particle. The specific $O(n+1,2)$ space-time conformal symmetry of the problem is obviously recovered as a group of symplectomorphisms of the space of motions.

BRS-Quantization of the "Energy" $\rho$. As in the previous example we use the vertical polarization $(Q=$ const.) and identify the Hilbert space of square integrable half-densities on $\mathbf{R}^{n+1,2}$ with $L^{2}\left(\mathbf{R}^{n+1,2}, d Q\right)$. Quantization of the constraint functions is fairly straightforward and we find:

$$
\begin{aligned}
\tau(\bar{Q} \cdot Q) f(Q) & =i \bar{Q} \cdot Q f(Q), \\
\tau(\bar{P} \cdot Q) f(Q) & =Q \cdot \frac{\partial f}{\partial Q}+\frac{n+3}{2} f(Q),
\end{aligned}
$$




$$
\tau(\bar{P} . \mathrm{P}) f(Q)=-i \Delta f(Q),
$$

where $\Delta$ is the Laplace-Beltrami operator of $\mathbf{R}^{n+1,2}$. Conspicuously $\tau$ realizes a homomorphism from $s l(2, \mathbf{R})$ to the skew self-adjoints of $L^{2}\left(\mathbf{R}^{n+1,2}\right)$; it is derived from the metaplectic representation restricted to the double covering of $\operatorname{Sl}(2, \mathbf{R})$ viewed as a subgroup of the metaplectic group. Using the following parametrization of $\mathbf{R}^{n+1,2} \backslash\{\mathbf{0}\}$,

$$
Q=R\left(\begin{array}{c}
x \\
\cos t \\
\sin t
\end{array}\right) \in \mathbf{R}^{n+1} \times \mathbf{R}^{2} \backslash\{0\}\left\{\begin{array}{l}
R \in \mathbf{R}_{+}^{*} \\
x \in \mathbf{R}^{n+1} \\
t \in[0,2 \pi)
\end{array}\right.
$$

one can cast the quantum constraints into the form

$$
\begin{gathered}
R^{2}\left(\|x\|^{2}-1\right) f(R, x, t)=0, \\
R \partial_{R} f+\frac{n+3}{2} \mathrm{f}(R, x, t)=0, \\
\sum_{j, k}\left(\delta^{j k}-x^{j} x^{k}\right) \partial_{j} \partial_{k} f-R \partial_{R} f+\sum_{j} x^{j} \partial_{j}\left(2 R \partial_{R} f-f\right)-R^{2} \partial_{R}^{2} f-\partial_{t}^{2} f=0,
\end{gathered}
$$

where $\partial_{j} f=\partial f / \partial x^{j}$ for $j=1 \cdots n+1$.

Writing $x=r u\left(r \in \mathbf{R}^{+}, u \in S^{n}\right)$ we find that the conditions (4.14) and (4.15) imply that $f$ is a distribution of the form $f(R, x, t)=R^{-(n+3) / 2} \delta(r-1) \hat{f}(u, t)$. The form is compatible with condition (4.16) which forces $\hat{f}$ to satisfy the PDE

$$
\left[\Delta_{S^{n}}-\frac{\partial^{2}}{\partial t^{2}}-\frac{(n-1)^{2}}{4}\right] \hat{f}(u, t)=0
$$

interpreted as the "conformally invariant" wave equation $\left[\Delta_{S^{n} \times S^{1}}-((n-1) / 4 n) R\right] \hat{f}=0$ for massless particles $\left(R=n(n-1)\right.$ is the scalar curvature of $\left.S^{n} \times S^{1}\right)$.

Unfortunately enough the solutions of this equation reduce to zero for $n$ even (the reduced Hilbert space is trivial in this case). Nevertheless, if $n$ is odd, one easily checks that the solutions of (4.17) are of the form $\hat{f}=\hat{f}_{+}+\hat{f}_{-}$with

$$
\hat{f}_{ \pm}(u, t)=\sum_{k \in N} e^{ \pm i t E_{k}} \tilde{f}_{k}^{ \pm}(u),
$$

where the $\tilde{f}_{k}^{ \pm}$'s are eigenfunctions of $\Delta_{S^{n}}$ with eigenvalues $-k(k+n-1)$ and

$$
E_{k}=k+\frac{n-1}{2}, \quad k \in N \text {. }
$$

The reduced Hilbert space thus splits as $\mathscr{H}_{\text {red }}=\mathscr{H}_{\text {red }}^{+} \oplus \mathscr{H}_{\text {red }}^{-}$and each subspace $\mathscr{H}_{\text {red }}^{ \pm}$can be identified with $L^{2}\left(S^{n}\right) \cong \sum_{k \in \mathbf{N}}^{\oplus} \mathbf{C}^{d(n, k)}$ if we drop the $t$-dependence in (4.18). The degeneracy is given by $d(k, n)=\left(\begin{array}{c}n+k \\ k\end{array}\right)-\left(\begin{array}{c}n+k-2 \\ k-2\end{array}\right)$. Direct quantization of $B$ with the vertical polarization on each component $\dot{T} S^{n}$ would have given 
$\mathscr{H}_{B}=L^{2}\left(S^{n}\right) \oplus L^{2}\left(S^{n}\right)$. The Hamiltonian $h=\|p\|$ of $B$ admits a natural representative in $C^{\infty}(M)$, namely the $S O(2)$ momentum mapping $H=\rho=\bar{P} . T . Q$ which is clearly $S l(2, \mathbf{R})$-invariant. The vertical polarization being $O(n+1,2)$-invariant, we readily find the operator

$$
\tau(H) f(Q)=(T Q) \cdot \frac{\partial f}{\partial Q}
$$

or, in the chosen coordinate system $\tau(\rho) f=\partial f / \partial t$, and thus if $n$ is odd

$$
S p \frac{1}{i} \tau_{\text {red }}^{ \pm}(h)= \pm\left\{\mathbf{N}+\frac{n-1}{2}\right\}
$$

because of (4.18) and (4.19). In doing so we recover (only in the case $n$ odd) the same spectrum and the same multiplicities as those found by Rawnsley [Raw] who used the canonical Kähler polarization of $\dot{T} S^{n}$ viewed as a complex null cone in $\mathbf{C}^{n+1}$.

Remark. Our procedure breaks down in the case $n$ even because the energy is necessarily integral in the previous Fourier decomposition. This drawback of the quantum reduction still remains to be well understood. On the other hand, there exists an obstruction to the pairing [Bla2] between the vertical polarization of $\dot{T} S^{n}$ and its images under the Hamiltonian flow of $h=\|p\|$. Hence, in this case, direct quantization of $h$ on the reduced symplectic manifold turns out to be intractable.

\section{Reduction by a Non-Unimodular Group}

Classical Reduction. The cotangent bundle $B=T^{*} S^{n}$ can also be obtained by a Marsden-Weinstein reduction from $M=T\left(\mathbf{R}^{n+1,1} \backslash\{0\}\right)$ by the neutral component $S_{0}$ of the subgroup $S$ of lower triangular matrices of $G=\operatorname{Sl}(2, \mathbf{R}) ; S_{0}$ is a Borel subgroup of $G$. We denote by s the Lie algebra of $S$. As a subgroup of $S l(2, \mathbf{R})$, the non-unimodular group $S_{0}$ acts symplectically on $(M, \omega)$ according to

$$
(P Q) \rightarrow(P Q)\left(\begin{array}{cc}
a & 0 \\
b & 1 / a
\end{array}\right) \quad\left(a \in \mathbf{R}_{+}^{*}, b \in \mathbf{R}\right),
$$

and the associated momentum mapping $\Psi: M \rightarrow{ }^{*}$ reads

$$
\Psi(P, Q)=(\bar{P} \cdot Q, \bar{Q} \cdot Q)
$$

where $\bar{V}$ is the covector associated to the vector $V$ by the standard Lorentz metric $g=\operatorname{diag}(1, \ldots, 1,-1)$ of $\mathbf{R}^{n+1,1}$. As in Sect. 4 the canonical symplectic form on $T^{*} \mathbf{R}^{n+1,1}$ is given on $T\left(\mathbf{R}^{n+1,1} \backslash\{0\}\right)$ by $\omega=d \theta$ with $\theta=\frac{1}{2}(\overline{\mathbf{P}} \cdot d Q-d \bar{P} \cdot Q)$. be

The $M W$ reduced symplectic manifold $B=C / S_{0}$ with $C=\Psi^{-1}(0)$ turns out to

$$
B \cong B_{+} \cup B_{-}, \quad B_{ \pm} \cong T^{*} S^{n}
$$


two copies of the (co)tangent bundle to the celestial sphere ${ }^{6} S^{n}$ with it canonical symplectic structure. We note that 0 is a regular value of $\Psi$.

The Lorentz group $O(n+1,1)$ acts symplectically on $(M, \omega)$ and the associated momentum mapping $\Phi: M \rightarrow o(n+1,1)^{*}$ is given by

$$
\Phi(P, Q)=P \cdot \bar{Q}-Q \cdot \bar{P} .
$$

In particular, the Hamiltonian of a boost is $H=\bar{P} . \Lambda . Q$, where $\Lambda=\left(\begin{array}{cc}0 & \beta \\ t_{\beta} & 0\end{array}\right) \epsilon$ $o(n+1,1)$ represents the generator of the boost $\beta \in \mathbf{R}^{n+1}$. Since the group action commutes with $S_{0}$, this function $H$ descends to $B$ as $h_{2}=\langle p, \beta\rangle$-see Sect. 3. This can be seen with the help of the following parametrization of $M$ :

$$
\begin{aligned}
& Q=R\left(\begin{array}{c}
q r \\
1
\end{array}\right), \quad\left\{\begin{array}{l}
R \in \mathbf{R}^{*} \\
r \in \mathbf{R}^{+} \\
q \in \mathbf{R}^{n+1} \\
\|q\|^{2}=1
\end{array}\right. \\
& P=\lambda Q+\frac{1}{R}\left(\begin{array}{l}
p \\
0
\end{array}\right), \quad\left\{\begin{array}{l}
\lambda \in \mathbf{R} \\
p \in \mathbf{R}^{n+1}
\end{array}\right.
\end{aligned}
$$

and thus $\left.\theta\right|_{C}=\langle p, d q\rangle$ descends to the reduced manifolds $B=B_{+} \cup B_{-}$(see (5.3)), the sign \pm corresponding to sign $(R)$.

Quantum Reduction. Again, we use the vertical polarization, and since all observables we want to quantize are at most linear in momentum, we can quantize them directly without using the BKS pairing and get

$$
\begin{gathered}
\tau(\bar{P} . Q) f(Q)=R \frac{\partial f}{\partial R}+\frac{n+2}{2} f(Q), \\
\tau(\bar{Q} \cdot Q) f(Q)=i \bar{Q} \cdot Q f(Q), \\
\tau(\bar{P} . \Lambda . Q) f(Q)=\langle q, \beta\rangle\left\{\left(1-r^{2}\right) \frac{\partial f}{\partial r}+r R \frac{\partial f}{\partial R}\right\}+\frac{1}{r}(\beta-q\langle q, \beta\rangle) \cdot \frac{\partial f}{\partial q} .
\end{gathered}
$$

In this case the reduced Hilbert space is given by the equations (see (2.15))

$$
\begin{aligned}
& \tau(\bar{P} \cdot Q) f(Q)=-f(Q), \\
& \tau(\bar{Q} \cdot Q) f(Q)=0 .
\end{aligned}
$$

In the right-hand side of Eq. (5.10) we recognize the term $-\frac{1}{2} \operatorname{Tr}\left(\operatorname{ad}\left(\xi_{1}\right)\right)$, where $\xi_{1}=\left(\begin{array}{rr}1 & 0 \\ 0 & -1\end{array}\right)$ is the Lie algebra generator of dilations in $s$.

By using the same technique as in the previous section, we find that the reduced Hilbert space associated with each component (distinguished by $\operatorname{sign}(R)$ ) of the

6 The projective null cone in Minkowski space-time $\mathbf{R}^{n+1,1}$ 
reduced symplectic manifold $\left(B, \omega_{B}\right)$ consists of (generalized) functions of the form

$$
f(R, r, q)=|R|^{-(n+4) / 2} \delta(r-1) \hat{f}(q) \quad\left(\hat{f} \in L^{2}\left(S^{n}\right)\right) .
$$

We thus get an identification $\mathscr{H}_{\text {red }} \cong \mathscr{H}_{B} \cong L^{2}\left(S^{n}\right) \oplus L^{2}\left(S^{n}\right)$. For the quantization of the boost Hamiltonian $h_{2}=\langle p, \beta\rangle$, we find the induced operator

$$
\tau_{\mathrm{red}}\left(h_{2}\right) \hat{f}(q)=(\beta-q\langle q, \beta\rangle) \cdot \frac{\partial \hat{f}}{\partial q}-\frac{n}{2}\langle q, \beta\rangle \hat{f}(q),
$$

which corresponds exactly to the one we obtained by direct quantization on $T^{*} S^{n}$ (see Sect. 3).

Discussion. We explicitly need the correction " $-\frac{1}{2} \operatorname{Tr}$ ad" in order to get the correct answer and to insure that quantization and reduction commute. This constitutes the first example known to us of a non-trivial application of the BRS-KS method applied to constrained Hamiltonian systems (see [Tuy2] for other examples).

\section{The Spin}

Introduction. The idea is now to handle the spin phase space $\left(B, \omega_{B}\right)=\left(S^{2}, s\right.$.Surf $)$ by means of a MW symplectic reduction with group $U(1)$. Here $s$ is a strictly positive real constant to be interpreted as the classical scalar spin [Sou1] and "Surf" denote the Riemannian surface element of the unit sphere $S^{2} \subset \mathbf{R}^{3}$. To this end we consider the "extended" manifold $M=C^{2} \backslash\{0\}$ endowed with the symplectic 2 -form ${ }^{7} \omega=d \theta$, where $\theta=1 / 2 i(\overline{\mathbf{Z}} \cdot d \mathbf{Z}-d \overline{\mathbf{Z}}$.Z $)$. The bar "—" stands for transposition plus complex conjugation. It is clear that $U(1)$ acts symplectically on $(M, \omega)$ according to $\mathbf{Z} \rightarrow \mathbf{Z} . e^{i \phi}$. This action is Hamiltonian and an associated momentum mapping $\Psi_{s}: M \rightarrow \mathbf{R} \cong u(1)^{*}$ is given by

$$
\Psi_{s}(Z)=\overline{\mathbf{Z}} . \mathbf{Z}-2 s .
$$

The constrained manifold of given (classical) spin $C_{s}:=\Psi_{s}^{-1}(0)$ is diffeomorphic to the 3 -sphere of radius $\sqrt{2 s}$ and the $\mathrm{MW}$ reduced manifold

$$
B=C_{s} / U(1) \cong S^{2}
$$

is the space of classical stationary states of spin $s=$ const. with symplectic 2-form $\omega_{B}=s$. Surf. The projection $C_{s} \rightarrow S^{2}: \mathbf{Z} \rightarrow \mathbf{u}$ is given explicitly by

$$
\sigma(\mathbf{u})=2 \frac{\mathbf{Z} \cdot \overline{\mathbf{Z}}}{\overline{\mathbf{Z}} \cdot \mathbf{Z}}-\mathbf{1},
$$

where $-i \sigma: \mathbf{R}^{3} \rightarrow s u(2)$ is the Lie algebra isomorphism realized by the Pauli matrices.

Prequantization. Again, prequantization of $(M, \omega)$ is readily achieved with the trivial principal bundle $P=M \times U(1)$ endowed with the connection 1-from

\footnotetext{
7 Twice the standard one!
} 
$\alpha=\theta+(d z / i z) \quad(z \in U(1))$ which can be written as $\alpha=(1 / i) \overline{\mathbf{Z}} . d \mathbf{Z}+\left(d z^{\prime} / i z^{\prime}\right)$ with $z^{\prime}=z \cdot \exp \left(-\frac{1}{2}|\mathbf{Z}|^{2}\right)$. A natural polarization $F$ is given in terms of the holomorphic complex 2 -form ${ }^{8} \varphi=d Z^{1} \wedge d Z^{2}$ by $F=\operatorname{ker}(\varphi)$. The Hilbert space $\mathscr{H}_{M}$ associated with $F$ consists of those half-forms $f^{\#}=f \otimes \sqrt{ } \varphi$, where $f: P \rightarrow \mathbf{C}$ is smooth and $F$-covariant constant, i.e. $D f \wedge \varphi=0$, where $D f=d f-$ if $\alpha$. Straightforward calculation then leads to $f(\mathbf{Z}, z)=z^{\prime} . \hat{f}(\mathbf{Z})$ with $\hat{f}$ holomorphic. The inner product of $\mathscr{H}_{M}$ is given by

$$
\langle f, g\rangle=\int_{M} \bar{f}(Z) \cdot \hat{g}(Z) \exp \left(-|\mathbf{Z}|^{2}\right) \lambda,
$$

where $\lambda$ denotes the Liouville 4-form of $(M, \omega)$-see also Sect. 3 .

Quantum Reduction. Quantizing the function $\Psi_{s}$ is straightforward. The associated skew self adjoint operator of $\mathscr{H}_{M}$ is plainly given by the $U(1)$-action lifted to the prequantum bundle, i.e.

$$
\tau\left(\Psi_{s}\right) \hat{f}=i(\mathscr{E}-2 s+1) \hat{f},
$$

where $\mathscr{E}=\sum_{a=1}^{2} Z^{a}\left(\partial / \partial Z^{a}\right)$ is the Euler vector field.

We now introduce the "quantum spin" $s_{Q}$ related to the classical spin $s$ by

$$
s_{Q}=s-\frac{1}{2}
$$

(see [Woo]) and it is easy to see that the reduced Hilbert space $\mathscr{H}_{\text {red }}^{s}$ is zero except when

$$
s_{Q} \in \frac{1}{2} \mathbf{N} \text {. }
$$

In that case $\hat{f} \in \mathscr{H}_{\text {red }}^{s}$ iff $\mathscr{E} \hat{f}=2 s_{Q} \hat{f}$, so $\mathscr{H}_{\text {red }}^{s}$ is the $2 s_{Q}+1$ dimensional Hilbert space of homogeneous holomorphic polynomials of degree $2 s_{Q}$.

Had we quantized $\left(B, \omega_{B}\right)=\left(S^{2}\right.$, s. Surf $)$ directly using the anti-holomorphic polarization on $S^{2} \cong \mathbf{P}_{1}(\mathbf{C})$, we would have obtained the same Hilbert space $\mathscr{H}_{\mathrm{B}} \cong \mathscr{H}_{\text {red }}^{s}$.

The Third Component of the Spin. Let us now BRS-KS quantize the following Hamiltonian $s_{3}=\left\langle\mathbf{s}, \mathbf{e}_{3}\right\rangle$ of $S^{2}$ (the "third component" of the spin vector $\mathbf{s}=s \mathbf{u}$ pointing in the direction $\mathbf{u} \in S^{2}$ ), represented on $M$ by the $U(1)$-invariant function,

$$
S_{3}=\frac{1}{2} \overline{\mathbf{Z}} \cdot \sigma_{3} \cdot \mathbf{Z} .
$$

Being a component of the $S U(2)$ momentum mapping, its quantization can be readily carried out because our polarization is $S U(2)$-invariant and

$$
\tau\left(S_{3}\right) \hat{f}=\frac{i}{2} \sum_{a=1}^{2}\left(\sigma_{3} \cdot \mathbf{Z}\right)^{a} \frac{\partial \hat{f}}{\partial Z^{a}}
$$

whose restriction to $\mathscr{H}_{\text {red }}^{s}$ yields the familiar spin representation: if $s_{Q}=n / 2$ we

\footnotetext{
${ }^{8}$ Every closed complex $n$-form $\varphi$ on a $2 n$-dimensional symplectic manifold $(M, \omega)$ defines a polarization iff $\operatorname{rank}(\varphi)=n$ and $\omega \wedge \varphi=0$
} 
readily find $\hat{f}(\mathbf{Z})=\sum \psi_{a_{1} a_{2} \cdots a_{n}} \cdot Z^{a_{1}} Z^{a_{2}} \cdots Z^{a_{n}}$ with $^{9} \psi_{a_{1} a_{2} \cdots a_{n}}=\psi_{\left(a_{1} a_{2} \cdots a_{n}\right)}$ and

$$
\tau_{\mathrm{red}}\left(s_{3}\right) \psi_{a_{1} a_{2} \cdots a_{n}}=\frac{i}{2} n \sum_{a_{0}=1}^{2} \sigma_{3}^{a\left(a_{1}\right.} \cdot \psi_{a_{2} a_{3} \cdots a_{n}} \widehat{a_{0}} .
$$

Note that direct quantization of $s_{3}$ on $\mathscr{H}_{B}$ would have given the same result. So, here again, geometric quantization is compatible with the BRS-KS reduction procedure.

The Spin Squared. The (SU(2)-Casimir) function $H=S_{1}^{2}+S_{2}^{2}+S_{3}^{2}$ is given by

$$
H(\mathbf{Z})=\frac{1}{4}(\overline{\mathbf{Z}} \cdot \mathbf{Z})^{2}
$$

whose restriction to $C_{s}$ descends to $B$ as the constant function $s_{1}^{2}+s_{2}^{2}+s_{3}^{2}=s^{2}$. Now this $H$ does not preserve the holomorphic polarization and thus must be quantized with the help of the infinitesimal pairing formula ${ }^{10}$ (see e.g. [Tuy1])

$$
\langle\tau(H) f, g\rangle=\int_{M}\left\{X_{H}(\bar{f}) \cdot g+\bar{f} \cdot g \frac{L_{X_{H}} \bar{\varphi} \wedge \varphi}{2 \lambda}\right\} \lambda,
$$

and leads after some technical computations to $\tau(H)=\frac{1}{4} \mathscr{E}^{2}+\frac{1}{2} \mathscr{E}$ whose restriction to $\mathscr{H}_{\text {red }}^{s}$ is the constant operator

$$
\frac{1}{i} \tau_{\text {red }}\left(s^{2}\right)=s_{Q}\left(s_{Q}+1\right) \mathbf{1} .
$$

Surprisingly enough, this corresponds to what is already known from "standard" quantum mechanics, although geometric quantization of the constant function $s^{2}$ on the reduced symplectic manifold $\left(S^{2}, s\right.$.surf $)$ would yield the constant operator: $s^{2} 1$. This should be taken as a success of the BRS-KS method.

Remarks. The star product (or Weyl-Moyal) BRS-quantization of the Casimir function $H$ does not lead to the "correct" previous result, the reason being that $\Delta^{2} H \neq 0$ (here $\Delta$ denotes the Laplace-De Rham operator on $\mathbf{C}^{2}$ ).

- In this example we see clearly that the choice of a representative influences the induced operator $\tau_{\text {red }}$. We could have represented the (constant) function $s^{2}$ on $B$ by the (constant) function $s^{2}$ on $M$, instead of by $H$. We then would have found $(1 / i) \tau_{\text {red }}\left(s^{2}\right)=s^{2} \mathbf{1}=\left(s_{Q}+(1 / 2)\right)^{2} \mathbf{1}$ instead of $s_{Q}\left(s_{Q}+1\right) \mathbf{1}$.

The Spin-Spin Interaction (e.g. the Fine Structure of the Hydrogen Atom). We consider now the spin phase space of a system of two particles $\left(B, \omega_{B}\right)=$ $\left(S^{2} \times S^{2}, s_{1}\right.$. Surf $_{1}+s_{2}$. Surf $\left._{2}\right)$ which can be obtained as a MW symplectic reduction of $(M, \omega)=\left(M_{1}, \omega_{1}\right) \times\left(M_{2}, \omega_{2}\right)=\left(\mathbf{C}^{2} \backslash\{\mathbf{0}\} \times \mathbf{C}^{2} \backslash\{0\}, \omega_{1}+\omega_{2}\right)$ with respect to the torus $U(1) \times U(1)$ and the momentum mapping $\left(\Psi_{s_{1}}, \Psi_{s_{2}}\right)$.

The Hilbert space we will deal with is obviously the one associated with the anti-holomorphic polarization $F=F_{1} \oplus F_{2}$, i.e. $\mathscr{H}_{M}=\mathscr{H}_{M_{1}} \otimes \mathscr{H}_{M_{2}}$. The reduced Hilbert space is thus $\mathscr{H}_{\text {red }}^{s_{1}, s_{2}}=\mathscr{H}_{\text {red }}^{s_{1}} \otimes \mathscr{H}_{\text {red }}^{s_{2}}$.

\footnotetext{
9 Round brackets denote symmetrization

$10 X_{H}$ denotes the Hamiltonian vector field associated with $H: L_{X_{H}} \alpha=0 \& \alpha\left(X_{H}\right)=H$
} 
Let us now quantize the Hamiltonian $h=\left\langle\mathbf{s}_{1}, \mathbf{s}_{2}\right\rangle$ on $B$ by viewing it as coming from the following $U(1) \times U(1)$-invariant function $H$ of $M$

$$
H\left(\mathbf{Z}_{1}, \mathbf{Z}_{2}\right)=\frac{1}{4} \sum_{j=1}^{3}\left(\overline{\mathbf{Z}}_{1} \cdot \sigma_{j} \cdot \mathbf{Z}_{1}\right) \cdot\left(\overline{\mathbf{Z}}_{2} \cdot \sigma_{j} \cdot \mathbf{Z}_{2}\right) .
$$

Some technicalities are needed to prove that, despite the fact that $F$ is not $H$-invariant, the pairing formula (6.10) yields the expected result (see (6.8))

$$
\tau_{\text {red }}(h)=\sum_{j=1}^{3} \tau_{\text {red }}\left(\left(s_{1}\right)_{j}\right) \otimes \tau_{\text {red }}\left(\left(s_{2}\right)_{j}\right) .
$$

Remark. In a previous paper dealing with the geometric quantization of the hydrogen atom [DET], we found a spurious coefficient in front of the spin-spin hyperfine Hamiltonian. This can be traced back to the fact that direct quantization of the classical Hamiltonian $h=\left\langle\mathbf{s}_{1}, \mathbf{s}_{2}\right\rangle$ on $\left(S^{2} \times S^{2}, \operatorname{Surf}_{1}+\operatorname{Surf}_{2}\right)$ gives $\frac{2}{9}$ times the result of (6.13). Here we have found the coefficient 1 which is felt as the correct one by physicists. Again, the BRS-KS technique seems to overcome several drawbacks of the geometric quantization scheme applied directly to constrained symplectic manifolds such as the spin phase spaces.

\section{Conclusion and Final Remarks}

We would start the discussion by mentioning that the overall impression of the compatibility between geometric quantization and the KS reduction procedure is quite favourable: in all but one case (see Sect. 4) we are able to identify the reduced Hilbert space $\mathscr{H}_{\text {red }}$ with a direct quantization $\mathscr{H}_{B}$ and using this identification, the operators $\tau_{\text {red }}(h)$ and $\tau_{B}(h)$ coincide in most cases.

However we have to admit that there remain several problems we have no answer for. Especially we do not have a theorem giving sufficient conditions for the above mentioned compatibility except the theorem of Gotay [Got]. Unfortunately the conditions of his theorem are quite restrictive, i.e. the action of the symmetry group $G$ is the canonical lift to the cotangent bundle of a free action on the configuration space, the group $G$ admits a bi-invariant metric and the observables to quantize are at most linear in the momentum variables. With the exception of the first example, none of our examples verifies these conditions, in particular those on the symmetry group.

In the first three examples we study $\dot{T}^{*} S^{n}$ as a reduced symplectic manifold. These reductions are related to different subgroups of $\operatorname{Sl}(2, \mathbf{R})$ and exhibit different groups of symplectomorphisms, i.e. the isometries $O(n+1)$ and the conformal transformations $O(n+1,1)$ of $S^{n}$. Moreover we may remark that $\dot{T} S^{n}$ is a coadjoint orbit of $O(n+1,1)$ and a component of a coadjoint orbit of $O(n+1,2)$ [Sou2]. We see that in these cases, reduction and geometric quantization commute when we use the vertical polarization although the method seems to break down in the second example: $T^{*} \mathbf{R}^{n+1,2}$ with $n$ even (see Sect. 4). However for $n$ odd we recover a result of Rawnsley [Raw] who obtained the spectrum of the square root $\|p\|$ of the kinetic energy with the help of a non-unitary pairing between a Kähler polarization and the vertical polarization of $\dot{T} S^{n}$. 
It should be stressed that the third example unexpectedly justifies the seemingly contradictory modification of the quantization of constraints by adding " $\frac{1}{2} \operatorname{Tr}$ ad" for skew-self adjoint operators. In the case of a lifted cotangent bundle action this fact can be explained by a consistent identification of the Hilbert space of $\frac{1}{2}$-densities on the reduced configuration space and a subspace (i.e. $\mathscr{H}_{\text {red }}$ ) of the Hilbert space of $\frac{1}{2}$-densities on the original configuration space [Tuy2]. The general case still remains to be elucidated.

In the last example we deal with the problem of spin Hamiltonians and recover the well known unitary irreducible (spin) representations of $S U(2)$. It is worth noticing that the method leads to a "correct" quantization of the total spin squared, i.e. if the eigenvalue of the operator associated to the classical total spin $s$ equals $s_{Q}$ then the eigenvalue of the operator associated to $s^{2}$ equals $s_{Q}\left(s_{Q}+1\right)$. This result cannot be obtained by quantizing the classical phase space $S^{2}$ directly. We also see that there is a difference between classical and quantum values of spin ( $\left.s=s_{Q}+\frac{1}{2}\right)$ which arises with the use of half-forms. This phenomenon is already discussed in [Woo, Sni]. Recently the same result has been obtained by using Feynman path integral quantization for spin [NR]. Fortunately enough reduction and geometric quantization do not always commute as we have seen in the example of the case of two spins (Sect. 6).

It should be mentioned that we have only considered here the very special case in which the symmetry group $G$ operates freely, more precisely in which $0 \in \mathscr{G}^{*}$ is a regular value (not only a weakly regular value) of the momentum mapping. Nevertheless we encountered several problems especially concerned with the construction of the reduced Hilbert space. We solved this difficulty in each case separately by enlarging the original Hilbert space to all functions (or even distributions) and then imposing the "natural" inner product on the reduced space. One might imagine that this can be justified by the spectral theorem.

Note added. After the completion of this article, we learned from M. Gotay (private communication) that in his approach [Got], one actually only needs the group to be unimodular. It also came to our attention that the problem we encounter in Sect. 4 for $n$ even is directly related to the obstruction for $p+q$ odd encountered in $[\mathrm{K} \phi]$ in constructing representations for $S O(p, q)$.

[KФ] Kostant, B., $\emptyset$ rsted, B.: 'The orbit method for minimal nilpotent coadjoint orbits, I.' (in preparation/private communication)

\section{References}

[Bla1] Blattner, R. J.: Pairing of half-form spaces. In: Coll. Int. CNRS n²37. Souriau, J. M. (ed.). pp. 175-186 Aix en Provence: CNRS 1974

[Bla2] __ The metalinear geometry of non-real polarizations, Lecture Notes in Math. Vol. 570, pp. 11-45. Berlin, Heidelberg, New York: Springer 1977

[BRS] Becchi, C., Rouet, A., Stora, R.: The abelian Higgs Kibble model, unitarity of the $S$-operator, Phys. Lett. B52, 344-346 (1974); Renormalization of gauge theories, Ann. Phys. (NY) 98, 287-321 (1976)

[Dir] Dirac, P. A. M.: Lectures on quantum mechanics, Belfer Graduate School of Science, Yeshiva University, New York (1964)

[Dub] Dubois Violette, M.: Systèmes dynamiques contraints: l'approche homologique. Ann. Inst. Fourier 37, 45-57 (1987) 
[DET] Duval, C., Elhadad, J., Tuynman, G. M.: Hyperfine interaction in a classical hydrogen atom and geometric quantization. J. Geom. Phys. 3, 401-420 (1986)

[Elh] Elhadad, J.: Quantification du flot géodésique de la sphère $\mathbf{S}^{\mathrm{n}}$, C.R. Acad. Sci. Paris 285 961-964 (1977)

[FHST] Fisch, J., Henneaux, M., Stasheff, J., Teitelboim, C.: Existence, uniqueness and cohomology of the classical BRST charge with ghosts of ghosts, Commun. Math. Phys. 120, 379-407 (1988)

[FV] Fradkin, E. S., Vilkoviski, G. A.: Quantization of relativistic systems with constraints, equivalence of canonical and covariant formalisms in quantum theory of gravitational field CERN Report Th. 2332 (1977)

[Got] Gotay, M. J.: Constraints, reduction, and quantization. J. Math. Phys. 27, 2051-2066(1986)

[GS] Guillemin, V., Sternberg, S.: Geometric Asymptotics. Providence, RI: American Mathematical Society 1977

[HT] Henneaux, M., Teitelboim. C.: BRST cohomology in classical mechanics. Commun. Math. Phys. 115, 213-230 (1988)

[Kos] Kostant, B.: Quantization and unitary representations. Lecture Notes in Mathematics, vol. 170, pp. 87-208 Berlin, Heidelberg, New York: Springer 1970

[KS] Kostant, B., Sternberg, S: Symplectic reduction, BRS cohomology, and infinite-dimensional Clifford algebras. Ann. Phys. (NY) 176, 49-113 (1987)

[Lol] Loll, R.: The extended phase space of the BRS approach. Commun. Math. Phys. 119, 509-527 (1988)

[MW] Marsden, J., Weinstein, A.: Reduction of symplectic manifolds with symmetry. Rep. Math. Phys. 5, 121-130 (1974)

[Mos1] Moser, J.: Regularization of Kepler's problem and the averaging method on a manifold, Commun. Pure Appl. Math. 23, 609-636 (1970)

[Mos2] __ : Various aspects of integrable Hamiltonian systems. In: Dynamical Systems. Guckenheimer, J., Moser, J., Newhouse, S. E. (eds.) Progress In Math. Vol. 8, Boston, MA: Birkhauser 1980

[NR] Nielsen, H. B., Rohrlich, D.: A path integral to quantize spin, Nucl. Phys. B299, 471-483 (1988)

[Raw] Rawnsley, J.: A nonunitary pairing of polarizations for the Kepler Problem. Trans. A.M.S. 250, 167-178 (1979)

[Sni] Sniatycki, J.: Geometric quantization and quantum mechanics. Appl. Math. Sci. Vol. 50, Berlin, Heidelberg, New York: Springer 1980

[Sou1] Souriau, J. M.: Structure des systèmes dynamiques. Paris: Dunod 1969

[Sou2] ___ Sur la variété Kepler. In: Symposia Mathematica XIV (1974), pp. 343-360; Géométrie globale du problème à deux corps. In: Proceedings IUTAM-ISIMM Modern Developments in Analytical Mechanics, Atti della Accademia delle Scienze di Tornio. Supp. al Vol. 117, 369-418 (1983)

[Sta] Stasheff, J.: Constrained poisson algebras and strong homotopy representation. Bull. AMS 19, 287-290 (1988)

[Tuy1] Tuynman, G. M.: Generalized Bergman kernels and geometric quantization. J. Math. Phys. 28, 573-583 (1987)

[Tuy2] __ : Reduction, quantization and non-unimodular groups. J. Math. Phys. 31, (1990)

[Woo] Woodhouse, N.: Geometric quantization. Oxford: Oxford University Press 1980

Communicated by A. Jaffe

Received May 11, 1989; in revised form July 5, 1989 
